\title{
Brief Cognitive Screening Battery (BCSB) is a very useful tool for diagnosis of probable mild Alzheimer's disease in a geriatric clinic
}

\author{
Bateria Breve de Rastreio Cognitivo (BBRC) para o diagnóstico da doença de Alzheimer \\ leve em uma clínica geriátrica \\ Helenice Fichman-Charchat', Cristina Vieira Mirandaํ', Conceição Santos Fernandes ${ }^{1}$, Daniel Mograbi', \\ Rosinda Martins Oliveira², Regina Novaes ${ }^{3}$, Daniele Aguiar ${ }^{3}$
}

\begin{abstract}
The diagnosis of early signs of Alzheimer's disease (AD) is a major challenge in a heterogeneous population. Objective: To investigate the use of the Brief Cognitive Screening Battery (BCSB) for the diagnosis of mild AD in a geriatric outpatient unit of a public hospital in the city of Rio de Janeiro. Method: BCSB was administered to 51 elderly adults with a clinical diagnosis of probable AD and 123 older adults without dementia (non-AD). Results: AD patients performed worse than non-AD group in all BCSB tests, except Clock Drawing $(p=0.10)$. The ROC curves and Logistic Regression analysis indicated that delayed recall in the figure memory test was the best predictor, screening mild AD patients with sensibility and specificity superior to $80 \%$. Conclusion: The BCSB was accurate in identifying people with AD in a geriatric outpatient clinic at a public hospital, including elderly people with chronic diseases, physical frailty and cognitive impairment
\end{abstract}

Keywords: Alzheimer's disease, aging, memory.

\section{RESUMO}

O diagnóstico de sinais precoces da doença de Alzheimer (DA) é um grande desafio. Objetivo: Investigar o uso da Bateria Breve de Rastreio Cognitivo (BCSB) para o diagnóstico de DA leve, em ambulatório de geriatria de hospital público do Rio de Janeiro. Método: A BCSB foi administrada a 51 idosos com DA e 123 idosos sem demência (não-DA). Pacientes DA tiveram pior desempenho do que o grupo não-DA na BCSB, exceto desenho do Relógio $(p=0,10)$. Resultados: Os resultados se mativeram após o controle de escolaridade e idade. As curvas ROC e análise de regressão logística indicaram que evocação tardia do teste de memória de figuras foi o melhor preditor para rastreio, com sensibilidade e especificidade superiores a 80\%. Conclusão: A BCSB, especialmente evocação tardia, identificou pessoas com DA em ambulatório de geriatria de hospital público, em uma amostra incluindo idosos com doenças crônicas, fragilidade física e comprometimento cognitivo.

Palavras-chave: doença de Alzheimer, envelhecimento, memória.

Neuropsychological and functional assessments are used to discriminate the transition between healthy aging and dementia $^{1,2}$. Brief cognitive and functional batteries may be used to screen for dementia, especially Alzheimer's disease $(\mathrm{AD})^{3,4,5,6}$. Most instruments have been validated and have norms constructed in developed countries, not adjusted to a heterogeneous socio-demographic and cultural as style a brazilian older adult population ${ }^{7,8,9,10,11,12,13,14}$. The Mini-Mental State Examination (MMSE) is the most widely used measure in research and clinical contexts ${ }^{12,13}$, This test is influenced by age and years of schooling ${ }^{12,13}$ making necessary the combination of MMSE with other memory, executive and attention functions tasks ${ }^{13,14,15}$. The Brief Cognitive Screening Battery (BCSB) developed by Nitrini et al. ${ }^{4}$ is one of the best assessments combining MMSE with other cognitive tasks has been used for detection of $\mathrm{AD}$ in Brazil and other developing countries with heterogeneous population profile in terms of clinical and demographic characteristics ${ }^{3,5,14,15,16,17}$.

The BCSB has been used in epidemiological and clinical research ${ }^{3,45,14,15,16,17}$. Radanovic et al. ${ }^{3}$ and Takada et al. ${ }^{6}$ demonstrated the high accuracy of this brief cognitive battery in the diagnosis of $\mathrm{AD}$ in illiterate, low, middle and high school

'Pontifícia Universidade Católica do Rio de Janeiro, Rio de Janeiro RJ, Brazil;

¿Universidade Federal do Rio de Janeiro, Rio de Janeiro RJ, Brazil:

${ }^{3}$ Hospital Federal dos Servidores do Estado do Rio de Janeiro, Rio de Janeiro RJ, Brazil.

Correspondence: Conceição Santos Fernandes;Avenida das Américas, 1245 / Apto 1507; 26310-000 Rio de Janeiro RJ, Brasil; E-mail: conceicaosf@yahoo.com.br

Conflic of interest: There is no conflict of interest to declare.

Received 26 March 2015; Received in final form 29 September 2015; Accepted 19 October 2015. 
levels. In another recent study using the same battery of tests, Charchat-Fishman et al..$^{5}$ describe a neuropsychological profile of mild cognitively impaired elderly assisted in a Geriatric outpatient clinical. The findings present a predominance of disexecutive mild cognitive impairment in patients with high vascular and metabolic risk. The aim of this study is to investigate the accuracy of the BCSB to screen mild Alzheimer's disease among elderly patients assisted in a geriatric outpatient clinic at a public hospital in the city of Rio de Janeiro. The patients included in this geriatric clinical group have cognitive and physical frailty, presence of chronic diseases and heterogeneous levels of education.

\section{METHOD}

\section{Sample}

The sample for this study consisted of older adults geriatric outpatients of a public hospital in the city of Rio de Janeiro. Fifty one elderly patients with clinically probable diagnosis of Alzheimer's dementia ${ }^{18}$ and 123 elderly without dementia were investigated. We adopted as the gold standard the diagnosis of dementia conducted by the medical staff of that service, which meets the diagnostic criteria of the NINCDS - ADRDA criteria of the American Psychiatric Association (APA), the DSM - IV - TR ${ }^{18}$ and the ICD- $10^{19}$.

The patients were referred by the health center for the geriatric clinic, which performed a screening with an evaluation protocol established by a team of professionals, consisting of a social worker, occupational therapist and geriatrician. The Inclusion criteria for care in the geriatrics department of the hospital are: a) more than 60 years of age, and b) to have at least two of the following clinical conditions: geriatric syndromes; dementia or mild cognitive impairment syndromes; disorders of mood and behavior; changes in speech and language; gait, or balance impairments or falls; severe sensory deficits; functional disability; urinary Incontinence. Were excluded from the elderly sample individuals with eyesight and hearing impairment without the use of correction, diagnosis of Parkinson's disease, head trauma brain injury (TBI), severe Alzheimer's disease, vascular dementia and mixed dementia.

\section{Ethical aspects}

This study was approved by the Research Ethics Committees of the University and of the hospital (000.320). People participated in this research by signing a term of informed consent, according to Resolution 196/96 of the National Health Council, which deals with the guidelines and standards for research involving humans.

\section{Instruments}

The tests were administered using standard stimuli. The record of the correct answers and observations were made with pencil and paper. The time was measured with the use of manual stop-watches. Neuropsychological tests were standardized and validated tasks to assess attention, episodic memory, semantic memory, visual spatial/constructive skills, executive functions and language. The $\mathrm{BCSB}^{3,4,5}$ included Mini-Mental State Examination, Animal Verbal Fluency, Clock Drawing and Figure memory tests (naming, incidental, immediate memory, learning, delay recall and recognition). The functional activities were assessment using Lawton scale that investigates the degree of independence in daily living tasks. There are four response options, numbered 0-3, with higher scores indicating greater disability. For more information, see Lawton and Brody ${ }^{20}$ and the activities of daily living- index by Katz et al. ${ }^{21}$. This scale assesses the functional capacity of the elderly. It is provides a list of six items that are hierarchically related and reflect the patterns of child development (example: dressing, bathing, until the self-regulation such as eating and elimination or excretion). Each item is scored from zero (0) for the independence to three (3) for total dependence.

\section{Procedures}

Neuropsychological tests were administered using standardized stimuli presented individually in a quiet and well-lit room, in the geriatric outpatient clinic of a public hospital in the state of Rio de Janeiro. The objectives and procedures of the study were explained by the researcher. The participant who voluntarily agreed to participate in the study signed a consent form. The evaluation was realized in just one session. All subjects underwent the same neuropsychological and functional research protocol.

\section{Data analysis}

Comparisons between means of demographic and clinical profile data were performed through the t test or chi-square test. ANOVA was conducted to test for differences between groups, followed by ANCOVA controlling for age and education differences. For all cognitive variables, the assumption of normality was tested with a Shapiro-Wilk tests. In a few cases (e.g. recognition), the distribution was not normal. Nevertheless, it has been shown that ANOVA is a robust procedure against deviations of normality ${ }^{22,23}$. In addition, the use of non-parametric tests (e.g. Mann-Whitney test) in these cases delivered similar results, so the original analysis is reported here. Pearson correlations were used to explore the relationship between age and education with BCSB scores.

ROC curves were used to evaluate the accuracy of each test and define cutoff points for each variable. A diagnosis model using the short battery was proposed from the logistic regression analysis. This model generated an equation that determined the probability for each individual to present clinically probable diagnosis of Alzheimer's dementia. These results classified the subjects and defined the sensitivity and specificity of the battery of neuropsychological tests for the diagnosis of $\mathrm{AD}$ in the studied sample. 
These data analysis were performed with the aid of statistical software Statistical Package for Social Sciences (SPSS) version 16.0. For all analysis the value of significance was set at $\mathrm{p}<0.05$.

\section{RESULTS}

The physical and cognitive frailty of the sample is described based on the analysis of 123 patients profiles: $29.5 \%$ with depression, 3.3\% had previous vascular stroke, $79.5 \%$ had systemic arterial hypertension, $23 \%$ presented with high level of cholesterol, $1.6 \%$ with cardiovascular disease, $17.2 \%$ with diabetes mellitus.

Table 1 present the demographic, general cognitive status and functional abilities of the sample. There were no differences for gender ( $p=0.80$ ) or years of schooling $(p=0.07)$, but there was a significant effect of age $(p=0.01)$. The age range was 57 to 102 years old, with mean $=79.57$ and standard deviation $(\mathrm{SD})=6.98$. The range of schooling was 0 to 16 years, with mean $=4.41$ and $\mathrm{SD}=3.37$.

The Pearson correlation analysis showed that all BCSB variables were negatively correlated with age $(\mathrm{p}<0.01)$, and Clock Drawing ( $\mathrm{r}=0.43, \mathrm{p}<0.01)$ and Verbal fluency $(r=0.24, p<0.01)$ and were positively associated with years of schooling.

Results for the ANOVA analysis are presented in Table 2 describing significant difference between $\mathrm{AD}$ and non- $\mathrm{AD}$ groups in all cognitive variables, except for Clock Drawing.
The same result was presented using ANCOVA adjusted by age and schooling.

ROC curves were used to evaluate the accuracy of each test. The cutoff points, sensibility and specificity for each BCSB variables were generated by ROC analysis (Figure) and presented on Table 3. Memory variables better discriminated the $\mathrm{AD}$ and non-AD groups, especially delayed recall for the Figure memory test.

A enter method was used during logistic regression analysis. The final model included all cognitive variable, but delay recall figure memory was the only one with significant coefficient ( $\mathrm{p}<0.05$ ). This model correctly classified $83 \%$ of cases, with $82.6 \%$ specificity and $84 \%$ sensitivity. The final model had a chi-square value of 77.5 (8), p < 0.01. The -2 Log likelihood was 129.163, with Cox \& Snell R Square of 0.364 and 0.520 Nagelkerke R.

The analysis of the classification errors showed that non-AD group classified as $\mathrm{AD}$ showed performance on memory variables below or near the cutoff tests. One of the non-AD elderly misclassified had a combination of advanced age (over 80 years), and low educational level (less than 4 years). Two of them were illiterate, one had hypertension, other had symptoms of hypothyroidism and depression diagnosis. Elderly people with $\mathrm{AD}$ classified as non-AD showed performance on memory tests near the cutoff (5 - 7). Two of them misclassified had hypothyroidism, four advanced age (above 80 years) associated to low educational level and one history of stroke.

Table 1. Demographic, cognitive and functional abilities description.

\begin{tabular}{|c|c|c|c|}
\hline & No-AD $(n=123)$ Mean (SD) & $A D(n=51)$ Mean (SD) & $p$-value \\
\hline Age & $78.6(7.3)$ & $81.3(5.7)$ & 0.012 \\
\hline Gender (W/M) & $100 / 23$ & $35 / 15$ & 0.080 \\
\hline year of schooling & $4.7(3.5)$ & $3.7(2.8)$ & 0.077 \\
\hline MMSE & $21.53(4.67)$ & $16.08(4.32)$ & $<0.001$ \\
\hline \multicolumn{4}{|l|}{ Lawton } \\
\hline Self report & $17.4(3.4)$ & $16.0(3.2)$ & 0.019 \\
\hline Informant reporting & $14.9(4.0)$ & $12.4(3.5)$ & 0.001 \\
\hline \multicolumn{4}{|l|}{ Katz } \\
\hline Self report & $17.2(1.3)$ & $17.2(1.3)$ & 0.771 \\
\hline Informant reporting & $16.4(2.4)$ & $15.6(2.7)$ & 0.119 \\
\hline
\end{tabular}

MMSE: mini mental state examination; SD: standard deviation.

Table 2. Mean and standard deviation of cognitive tests.

\begin{tabular}{lccc}
\hline Variables & No-AD & AD & p-value* \\
\hline Incidental memory & $4.58(1.66)$ & $2.92(1.85)$ & $<0.001$ \\
Immediate memory 1 & $6.70(1.79)$ & $4.16(2.16)$ & $<0.001$ \\
Immediate memory 2 & $7.2(2.0)$ & $4.5(2.5)$ & $<0.001$ \\
Delayed recall test & $6.47(2.22)$ & $2.76(2.53)$ & $<0.001$ \\
Recognition & $8.93(1.97)$ & $6.82(2.91)$ & $<0.001$ \\
Clock Drawing & $4.67(2.37)$ & $3.62(2.51)$ & 0.066 \\
Animal Verbal Fluency & $11.82(4.03)$ & $7.86(3.26)$ & $<0.001$ \\
\hline F
\end{tabular}


Table 3. Cutt-off scores of the most discriminatives tests for sample.

\begin{tabular}{lcccc}
\hline & Area under the curve & Cut-off scores & Sensitivity (\%) & Specificity (\%) \\
\hline Delayed recall test & 0.867 & $<5$ & 71.9 & 88.1 \\
Immediate memory & 0.825 & $<5$ & 75 & 81 \\
Learning & 0.820 & $<5$ & $<8.1$ & 69 \\
Recognition & 0.766 & $<9$ & 61 & 69 \\
Verbal fluency & 0.749 & $<3$ & 71.9 & 73.8 \\
Incidental memory & 0.737 & $<3$ & 62.5 & 66.7 \\
Clock-drawing & 0.608 & & 57.1 \\
\hline
\end{tabular}

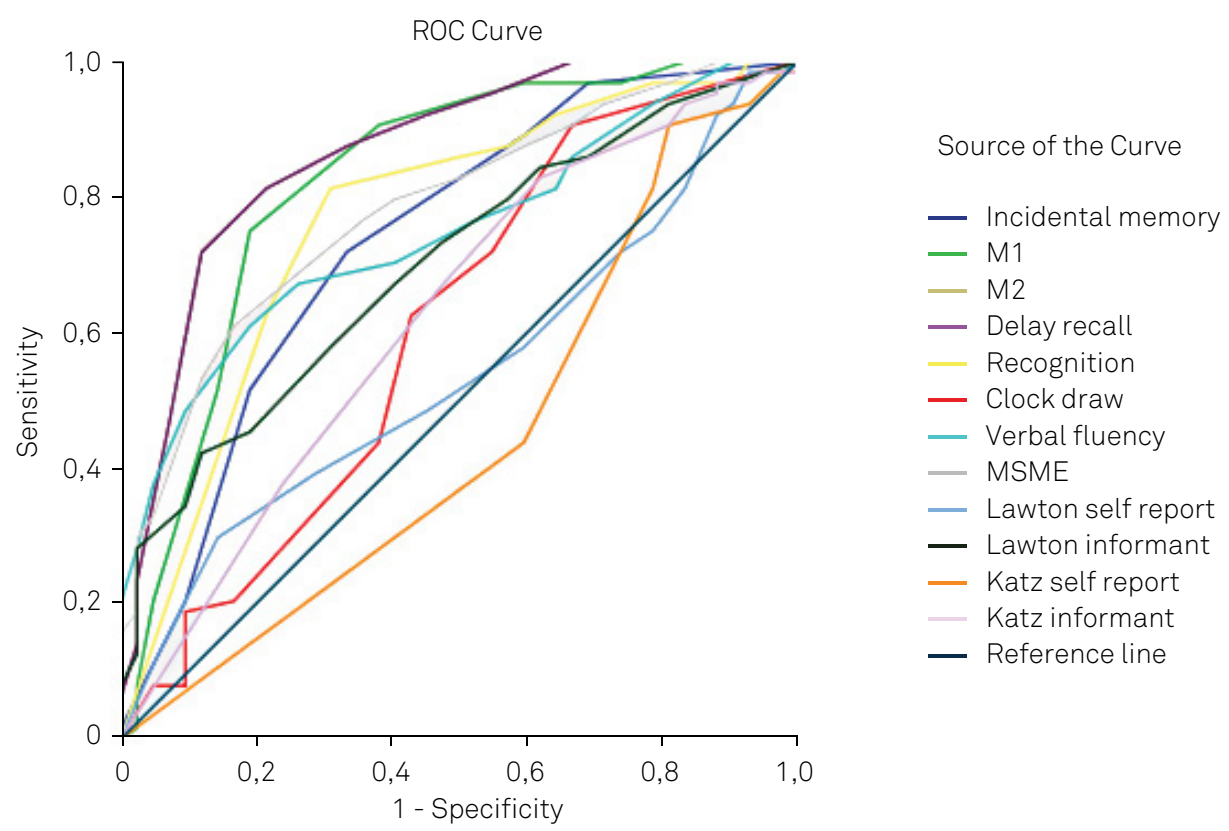

Figure. Diagonal segments are produced by ties.

\section{DISCUSSION}

The BCSB is accurate in identifying persons with Alzheimer's disease in a geriatric outpatient medical clinic of a public hospital.

\section{The sample}

The profile of the sample demonstrates cognitive, physical and demographic heterogeneity that creates challenges for cognitive screening and early identification of $\mathrm{AD}$. It includes persons with chronic diseases, depression symptoms and heterogeneity of age and educational level. This sample is very interesting because it reproduces the real life settings experienced by specialists or geriatricians at general medical clinics.

\section{The sensitivity and specificity of BCSB}

The BCSB sensitivity in this study was relatively lower than what was found for patients from a neurology outpatient clinic or in the community ${ }^{4,6,14}$. Vittielo et al. ${ }^{14}$ compared the neurological patients with selected healthy elderly as non-cases, different to the geriatric participant of the present study. Our sample included elderly with metabolic and psychiatric disorders such as hypertension (79.5\%), diabetes mellitus (17.2\%) and depression (29.5\%) that may affect cognition secondarily.

The relatively lower specificity of BCSB in our study may arise from the heterogeneity of education (range 0-16 years) and age (range 57-102 years) of the sample. There was a trend pointing to a difference between group in terms of schooling $(p=0.07)$, but no difference regarding age $(p=0.12)$. Epidemiological studies of AD using the BCSB confirmed these results showing that age and education are risk factors for $\mathrm{AD}^{15,24}$.

In this study all the variables of the BCSB are influenced by age. However, only executive function (Verbal Fluency and Clock Drawing) was related to schooling. These results showed again that BCSB memory testing measurements suffer little influence of education. Other previous studies found similar results ${ }^{3,6,14}$.

Furthermore, the possible inclusion of mild cognitive impairment $(\mathrm{MCI})$ patients in the non-AD group may have 
influenced the decrease in sensitivity and specificity of BCSB. Part of this sample was characterized previously by Charchat et al..$^{5}$ using the BCSB. They pointed out that most of the subjects were classified with a dysexecutive MCI, condition known as a possible intermediate between normal cognitive decline of aging and dementia process ${ }^{25}$. This might explain why functional variables and Clock Drawing test did not discriminate $\mathrm{AD}$ and non- $\mathrm{AD}$ groups.

\section{The ROC curve}

The non- $\mathrm{AD}$ subjects presented cognitive impairment especially in executive functions. In these patients, the area under the ROC curve for the Clock Drawing test was very low (0.61), demonstrating the inability of the BCSB to differentiate $\mathrm{AD}$ from non- $\mathrm{AD}$ groups.

In the other tasks of executive functions such as incidental memory, verbal fluency, the areas were between 0.70 and 0.80 , showed low discriminative ability. Similar results were described in Charchat-Fichman et al. ${ }^{5}$, using BCSB in non-AD subjects.

The areas under the ROC curve of BCSB tests had values above 0.70, except for the Clock Drawing test.

The variables of anterograde episodic memory (delayed recall, immediate memory and learning) showed the greatest ability to discriminate the group with $\mathrm{AD}$ and non- $\mathrm{AD}$, with areas under the ROC curve above 0.80 . This result was confirmed by logistic regression analysis that showed delayed recall as the main variable in the model. This model could predict $83 \%$ of cases.

Takada et al. $2006^{6}$ compared the accuracy of delayed recall task of BCSB in relation to the CERAD list of words. The accuracy of the BCSB to identify $\mathrm{AD}$ patients was higher than CERAD, especially in a sample of illiterate subjects.

\section{The performance in $A D$ group and in non-AD group}

The ANOVA followed by ANCOVA analysis adjusted by age and schooling, showed that the $\mathrm{AD}$ group had worse performance in all BCSB tasks, except Clock Drawing. The ROC and regression analysis pointed to the memory tasks, particularly delayed recall and learning, as the best to identify individuals with $\mathrm{AD}$ in a geriatric sample. These results are in agreement with previous clinical validation studies with the BCSB, that indicated recent episodic memory impairment as a hallmark of $\mathrm{AD}^{4,6,14,17}$. Studies with other brief batteries are in agreement that associative learning tasks and free delay recall are the best measures to detect mild and pre-clinical $\mathrm{AD}^{5,25,26,27}$, with changes in naming tasks, verbal fluency and semantic memory being usually found in more advanced stages of the disease $e^{5,25,26,27}$.

In conclusion, the BCSB is accurate in identifying persons with Alzheimer's disease in a geriatric outpatient medical clinic.

The present study may have strong clinical application because the cutoffs and sensitivity indexes can be used in other outpatient geriatric clinics as a reference for tracking Alzheimer's disease.

Recent episodic memory tests, specially delayed recall, showed greater ability to identify elderly patients with probable AD. Memory assessment in the context of a comprehensive geriatric assessment seems to be essential.

The BCSB is easy, fast and with low influence of educational level. The present study recommended the BCSB use for public ambulatory settings with high flow of elderly patients.

\section{References}

1. Paula JJ, Shlottdeldt CG, Moreira L, Cotta M, Bicalho MA, RomanoSiva MA et al. Psychometric properties of a brief neuropsychological protocol for use in geriatric populations. Rev Psiquiatr Clín. 2010;37(6):246-50. doi:10.1590/S0101-60832010000600002

2. Winblad B, Palmer K, Kivipelto M, Jelic V, Fratiglioni L, Wahlund LO et al. Mild cognitive impairment: beyond controversies, towards a consensus: report of the International Working Group on Mild Cognitive Impairment. J Int Med. 2004;256(3):240-6. doi:10.1111/j.1365-2796.2004.01380.x

3. Radanovic M, Carthery MT, Charchat H, Herrera JR, Porto SP, Nitrini R. Brief cognitive battery in the diagnosis of mild Alzheimer's disease in subjects with medium and high levels of education. Demen Neuropsychol. 2007;1:32-6.

4. Nitrini R, Lefèvre BH, Mathias SC, Caramelli P, Carrilho PE, Sauaia N et al. [Neuropsychological tests of simple application for diagnosing dementia]. Arq Neuropsiquiatr. 1994;52(4):457-65. Portuguese. doi:10.1590/S0004-282X1994000400001

5. Charchat-Fishman H, Fernandes C, Oliveira R, Caramelli P, Aguiar D, Novaes R. Predomínio de comprometimento cognitivo leve disexecutivo em idosos atendidos no ambulatório de geriatria de um hospital terciário na cidade do Rio de Janeiro. Neuropsicol Latinoam. 2013;5:31-40.

6. Takada LT, Caramelli P, Fichman HC, Porto CS, Bahia VS, Anghinah $\mathrm{R}$ et al. Comparison between two tests of delayed recall for the diagnosis of dementia. Arq Neuropsiquiatr. 2006;64(1):35-40. doi:10.1590/S0004-282X2006000100008

7. Spreen O, Strauss E. A compendium of neuropsychological tests: administration, norms, and commentary. 2nd ed. New York: Oxford University Press; 1998.

8. Mitrushina MN, Boon KB, D'Elia LF. Handbook of normative data for neuropsychological assessment. New York: Oxford University Press; 1999.

9. Sunderland T, Hill JL, Mellow AM, Lawlor BA, Gundersheimer J, Newhouse PA et al. Clock drawing in Alzheimer's disease: a novel measure of dementia severity. J Am Geriatr Soc. 1989;37(8):725-9. doi:10.1111/j.1532-5415.1989.tb02233.x

10. Prince M, Acosta D, Chiu H, Scazufca M, Varghese M. Dementia diagnosis in developing countries: a cross-cultural validation study. Lancet. 2003;3619(361):909-17. doi:10.1016/S0140-6736(03)12772-9

11. Plassman BL, Langa KM, Fisher GG, Heeringa ST, Weir DR, Ofstedal $\mathrm{MB}$ et al. Prevalence of cognitive impairment without dementia in the United States. Ann Intern Med. 2008;148(6):427-34. doi:10.7326/0003-4819-148-6-200803180-00005

12. Folstein MF, Folstein SE, McHugh PR. "Mini-mental state": a practical method for grading the cognitive state of patients for the clinician". J Psychiatr Res. 1975;12(3):189-98. doi:10.1016/0022-3956(75)90026-6 
13. Brucki SMD, Nitrini R, Caramelli P, Bertolucci PHF, Okamoto $\mathrm{IH}$. Suggestions for utilization of the Mini-Mental State Examination in Brazil. Arq Neuropsiquiatr. 2003;61(3B):777-81. doi:10.1590/S0004-282X2003000500014

14. Vitiello APP, Ciríaco JGM, Takahashi DY, Nitrini R, Caramelli P. Avaliação cognitiva breve de pacientes atendidos em ambulatório de neurologia geral. Arq Neuropsiquiatr. 2007;65(2A):299-303. doi:10.1590/S0004-282X2007000200021

15. Herrera EJr, Caramelli P, Silveira ASB, Nitrini R. Epidemiologic survey of dementia in a community-dwelling Brazilian population. Alzheimer Dis Assoc Disord. 2002;16(2):103-8. doi:10.1097/00002093-200204000-00007

16. Herrera E Jr, Caramelli P, Nitrini R. [Population epidemiologic study of dementia in Catanduva city: state of Sao Paulo, Brazil]. Rev Psiq Clín 1998;25:70-73. Portuguese.

17. Novaretti TMS, Radanovic M, Nitrini R. Screening for cognitive impairment in late onset depression in a Brazilian sample using the BBRC-EDU. Dement Neuropsychol. 2012;6(2):85-90.

18. American Psychiatric Association. DSM-IV-TR - manual diagnóstico e estatístico de transtornos mentais. 4a ed. Porto Alegre: Artmed; 2002.

19. World Health Organization - WHO. The ICD-10 classification of mental and behavioral disorders: clinical descriptions and diagnostic giuidelines. Geneve: World Health Organization; 2009.

20. Lawton MP, Brody EM. Assesment of older people: self-aintaining and instrumental activities of daily living. Gerontologist. 1969;9(3 Part 1):179-85. doi:10.1093/geront/9.3_Part_1.179
21. Katz S, Ford AB, Moskowitz RW, Jackson BA, Jaffe MW. Studies of illness in the aged. The index of ADL: a standardized measure of biological an psychosocial function. JAMA. 1963;185(12):914-9. doi:10.1001/jama.1963.03060120024016

22. Maxwell, S E, Delaney, H D. Designing experiments and analyzing data: a model comparison perspective. 2nd ed. London: Routledge Academic; 2003.

23. Sokal RR, Rohlf FJ. Biometry: the principles and practice of statistics in biological research. 3rd ed. New York:W. H. Freeman; 1995.

24. Nitrini R, Bottino CM, Albala C, Custodio Capuñay NS, Ketzoian C, Llibre Rodriguez JJ et al. Prevalence of dementia in Latin America: a collaborative study of populationbased cohorts. Int Psychogeriatr. 2009;21(4):622-30. doi:10.1017/S1041610209009430

25. Petersen RC, Smith G E, Waring SC, Ivnik RJ, Tangalos EG, Kokmen E. Mild cognitive impairment: clinical characterization and outcome. Arch Neurol. 1996;56(3):303-8.

26. Fischer P, Jungwirth S, Zehetmayer S, Weissgram S, Hoenigschnabl $\mathrm{S}$, Gelpi E et al. Conversion from subtypes of mild cognitive impairment to Alzheimer dementia. Neurology. 2007;68(4):288-91. doi:10.1212/01.wnl.0000252358.03285.9d

27. Petersen RC, Roberts RO, Knopman DS, Boeve BF, Geda YE, Ivnik RJ et al. Mild cognitive impairment: ten years later. Arch Neurol. 2009;66(12):1447-55. doi:10.1001/archneurol.2009.266 